\title{
Anatomy, pigmentation, ventral and dorsal subpopulations of the substantia nigra, and differential cell death in Parkinson's disease
}

\author{
W R G Gibb, A J Lees
}

\begin{abstract}
In six control subjects pars compacta nerve cells in the ventrolateral substantia nigra had a lower melanin content than nerve cells in the dorsomedial region. This coincides with a natural anatomical division into ventral and dorsal tiers, which represent functionally distinct populations. In six cases of Parkinson's disease (PD) the ventral tier showed very few surviving nerve cells compared with preservation of cells in the dorsal tier. In 13 subjects without PD, but with nigral Lewy bodies and cell loss, the degenerative process started in the ventral tier, and spread to the dorsal tier. This pattern of selective degeneration of nigrostriatal neurons is not seen in ageing or after acute administration of MPTP (1-methyl-4-phenyl-1,2,3,6tetrahydropyridine).
\end{abstract}

The most prominent pathological change in Parkinson's disease (PD) is degeneration of melanin pigmented brainstem nuclei. Consequently neuromelanin has been considered of potential importance in its pathogenesis. The main counter-argument is that nonpigmented regions such as the nucleus basalis, cerebral cortex, and parasympathetic nervous system may be damaged, whereas the pigmented arcuate and periventricular nuclei of the hypothalamus are spared. Melanin cannot therefore be critical to the degenerative process, although its formation from dopamine by autoxidation could release free radicals, and it is capable of binding toxic compounds. Additionally, demelanisation of the substantia nigra (SN) in PD is believed to result from selective death of the more heavily pigmented neurons. ${ }^{1}$ The nigral toxin MPTP ( 1 - m e th y $1-4-$ ph en y $1-1,2,3,6$ - te trahydropyridine), which causes a Parkinsonian syndrome in primates, destroys those nigral neurons containing the most melanin pigment, ${ }^{2}$ thus providing a possible analogy with PD.

We have examined the internal anatomy and regional variations in neuronal melanin in the normal human substantia nigra and compared this to patients with PD.

\section{Material and methods}

The $\mathrm{SN}$ from six controls (aged 55-86 years, median 75 years), six cases of PD (aged 61-87 years, median 69 years), and 13 persons without PD but with Lewy bodies in the $S N$, known as incidental Lewy body disease or presymptomatic $\mathrm{PD}^{3}$ (aged 50-87 years, median 77 years) were examined. The incidental cases showed Lewy bodies and mild nerve cell loss in the $\mathrm{SN}$ pars compacta, as well as in the locus coeruleus. They showed more severe nigral cell degeneration than is normal for ageing, nigral cell loss intermediate between normal and PD, and neuronal inclusions (Lewy bodies and pale bodies) identical to those of PD. Dopamine depletion is known to be present at the time of onset of PD, and such cases were presumed to correspond to a presymptomatic phase of the disorder. The purpose of studying them was to examine cell populations of the $\mathrm{SN}$ pars compacta at different stages of degeneration. Three of the incidental cases also had Alzheimer's disease verified by pathological examination.

For each brain the cerebral hemispheres were divided mid-sagittally and the brainstem divided parasagittally. This provided one half of the SN and the ventral tegmental area for histological study. The half brain was placed in $10 \%$ neutral formol saline for at least four weeks, and the brainstem was then cut perpendicular to its axis, from the emerging oculomotor nerve fibres to the lower border of the superior colliculus. Two $4 \mathrm{~mm}$ thick blocks of the lower midbrain were taken from the caudal segment of brainstem, and two similar blocks from the rostral segment. These were processed and serial $7 \mu \mathrm{m}$ or $14 \mu \mathrm{m}$ sections, cut in the transverse plane, were prepared. In half the cases every 10th and 11 th sections were stained, and in the rest every 24th and 25th. Haematoxylin and eosin (H\&E) was used to show cell populations and melanin, and luxol fast blue-cresyl violet (LFB-CV) to show cells and myelin. A Kontron IBAS 2000 image analyser was used to determine the surface area of cells occupied by melanin in subpopulations of the pars compacta in three cases. The system consists of a CCD camera attached to a Zeiss Axioplan microscope with an automated stepping stage. In regions of interest lines were traced around 100 cells and around the melanin contained in each one. Tyrosine hydroxylase antibody (Pel-Freez Biologicals, Arkansas) was used on sections from normal subjects using an avidinbiotin system. in revise.

Accepted 5 October 1990
King's College S Dentistry, London Nervous Diseases, Correspondence Department of Neurology, Institute of Psychiatry, 8AF, UK.

Received 16 March 1990 and 
Figure 1a-h Drawings of the $S N$ and paranigral nucleus at levels 1-8. The dots represent pigment deposits within nerve cells, thus, they are smallest in the ventrolateral group and small in the

paranigral nucleus. The

drawings were obtained by superimposing

photographic tracings to show the more consistent distribution patterns without quantitation relevant to section thickness. Cells were divided into arbitrary groups for the purposes of description. Levels 1 and 2 contain the rostral group, levels 3-6 the

intermediate, dorsal and ventrolateral groups, and levels 7 and 8 the caudal group. The paranigral nucleus is present at all levels. MB mamillary body, PN paranigral nucleus, CP cerebral peduncle, $P R$ pars reticulata, $R N$ red nucleus, $R G$ rostral group, $I G$ intermediate group, $D G$ dorsal group, $V L$ ventrolateral group, $P L$ pars lateralis, CG caudal group, $C D$ decussation of the superior cerebellar peduncle.
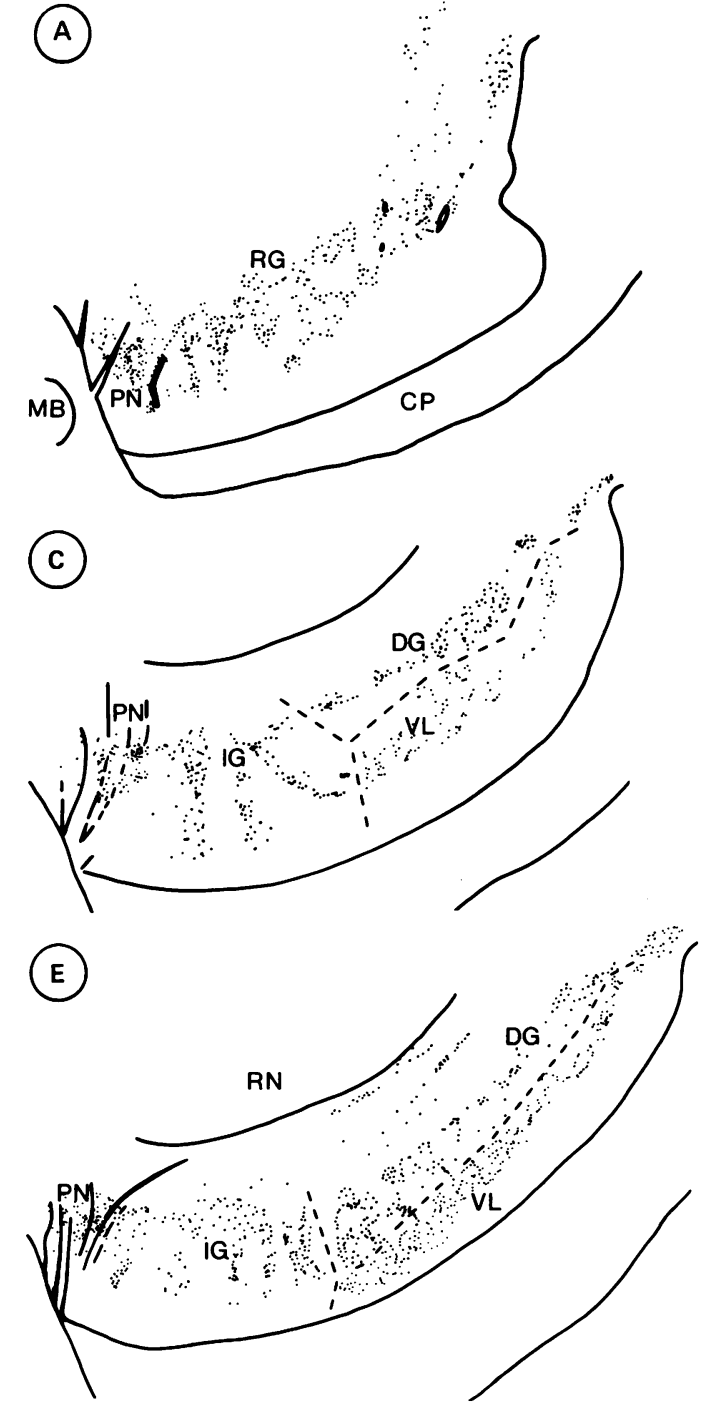

(G)

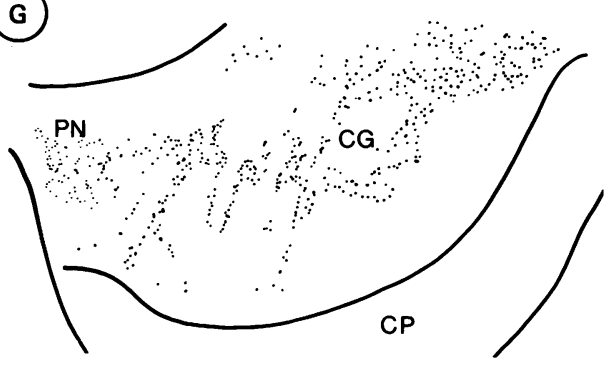

Results

Normal anatomy of the substantia nigra

The $\mathrm{SN}$ and paranigral nucleus were examined from the level of the mammillary nuclei to the ventral pontine nuclei. Descriptions of the pigmented central linear and intracapsular nuclei, and of the pedunculopontine nucleus are excluded. Considerable heterogeneity of cell numbers and packing density between cases was found and only the consistent anatomical features will be described. Scattered pigmented nerve cells appeared at the level of the mammillary nuclei representing the rostral limit of the substantia nigra. With caudal progression the cell population increased to form the rostral cell group of the pars compacta. A small cluster of closely packed cells lying caudal to the mammillary
(B)

nuclei and at the medial extent of the SN formed the paranigral nucleus (level 1, fig la).

At the rostral part of the red nucleus cell density and clumping was often accentuated by interpenduncular branches of the terminal basilar and posterior cerebral arteries passing through the pars compacta, thus dividing the medial nerve cells into groups which extended ventrally in columns (level 2, fig lb).

In later sections rostral fascicles of the oculomotor nerve followed a similar course through the paranigral nucleus and medial SN. The rostral cell group was replaced by intermediate, dorsal and ventrolateral cell groups (level 3, fig 1c). Cells of the intermediate group were arranged in small clumps with linear arrays projecting ventrally into the pars reticulata. The central to lateral pars compacta 


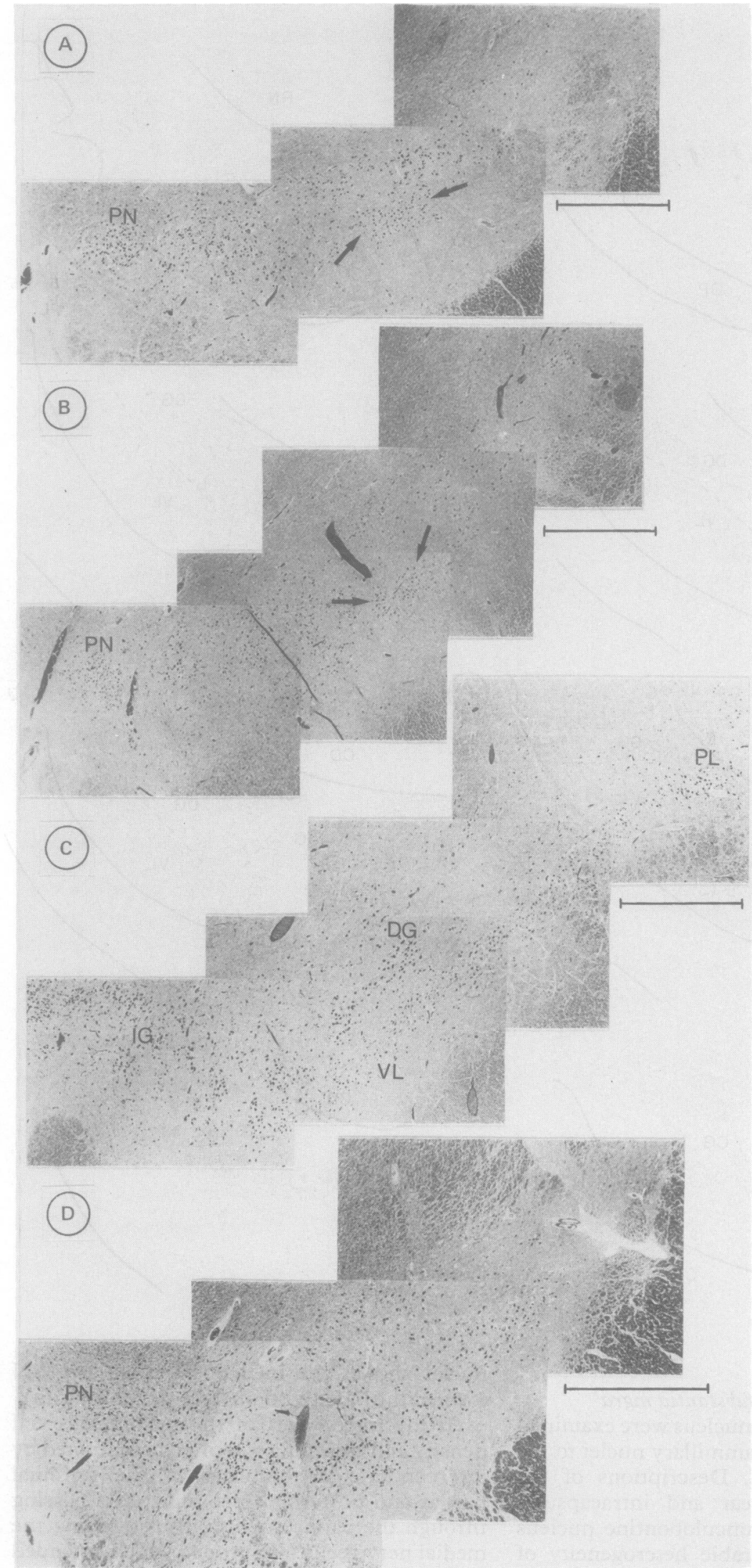

Figure 2 Horizontal sections of the $S N(14 \mu \mathrm{m})$ stained with HEE. a) Level 2, but without arterial perforators present medially. Despite the level there is an early clump of cells of the ventrolateral group (arrows). b) Level 2. This section also shows early cell clumps of the ventrolateral group (arrows). c) Level 5.d) Level 7. PN paranigral nucleus, IG intermediate group, DG dorsal group, VL ventrolateral group, PL pars lateralis. Scale equivalent to $2 \mathrm{~mm}$.

formed the dorsal cell group, with occasional columns of cells linking with the ventrolateral group. The most lateral region was the pars lateralis.
Fascicles of the oculomotor nerve and cells of the paranigral nucleus and pars compacta increased. Pigmented cells appeared along the capsule of the red nucleus forming a scattered line of cells posterior to the dorsal group. Thus there were three parallel populations, the ventrolateral group becoming the more dominant. Dorsal and ventrolateral groups were often angulated towards each other laterally so that they impinged on one another (level 4, fig 1d). The depth and breadth of the pars compacta was greatest at the mid-SN level (level 5, fig 1e).

In the low midbrain the interpenduncular fossa became shallow and the paranigral nucleus shifted towards the midline. The red nucleus disappeared and fibres of the superior cerebellar peduncle passed dorsal to the SN, decussating across the midline. Pigmented cells lay beside ventral fibres of the superior cerebellar peduncle (level 6, fig 1f). The ventrolateral group faded, and cells of the lateral SN were diffusely spread (level 7 , fig $1 \mathrm{~g}$ ).

Nerve cells moved dorsally to form a single strip along the superior cerebellar peduncle, termed the caudal cell group. The paranigral nucleus was smaller, and pontine nuclei developed at the medial and dorsal part of the cerebral peduncle (level 8, fig $1 \mathrm{~h}$ ).

The pars reticulata lay ventral to the pars compacta and increased in size to level 3. At lower levels its lateral part was reduced by the ventrolateral group and by a dorsal shift of the cerebral peduncle. The medial part continued to level 7 when it was eroded by the developing pons. The pars reticulata contained a few nonpigmented cells recognised by their prominent Nissl substance and nerve cell processes.

\section{Normal distribution of melanin in the substantia} nigra

The rostral group at levels 1 and 2 contained cells of similar morphological type and pigment intensity. The cells were deeply pigmented, often to the extent of obscuring the nucleus. Some cases showed occasional clumps of lightly melanised cells in the ventral or lateral $\mathrm{SN}$, with their long axes lying parallel in the same medial to lateral plane (figs $2 a$ and $b$ ).

At levels 3-6 the intermediate and dorsal groups retained the same deep pigment intensity as the rostral group. Pigmented cells in dorsal parts of the $\mathrm{SN}$ and the pars lateralis were heavily pigmented. In contrast cells of the ventrolateral group were lightly melanised (fig 2c). Light and dark cells were often seen juxtaposed where columns of cells bridged the dorsal and ventrolateral groups (figs $3 a$ and $b$ ). Laterally ventrolateral and dorsal groups were angulated towards each other and light and dark cell clusters lay adjacent to each other (figs $1 d$ and e). At levels 4, 5 and 6, seven pigmented cells of the dorsal group lying beside the red nucleus and cerebellar peduncle were heavily melanised. At levels 7 and 8 the caudal group contained heavily pigmented cells, as in the dorsal and intermediate groups (fig $2 \mathrm{~d}$ ).

The anatomically distinct population of lightly pigmented cells of the ventrolateral 


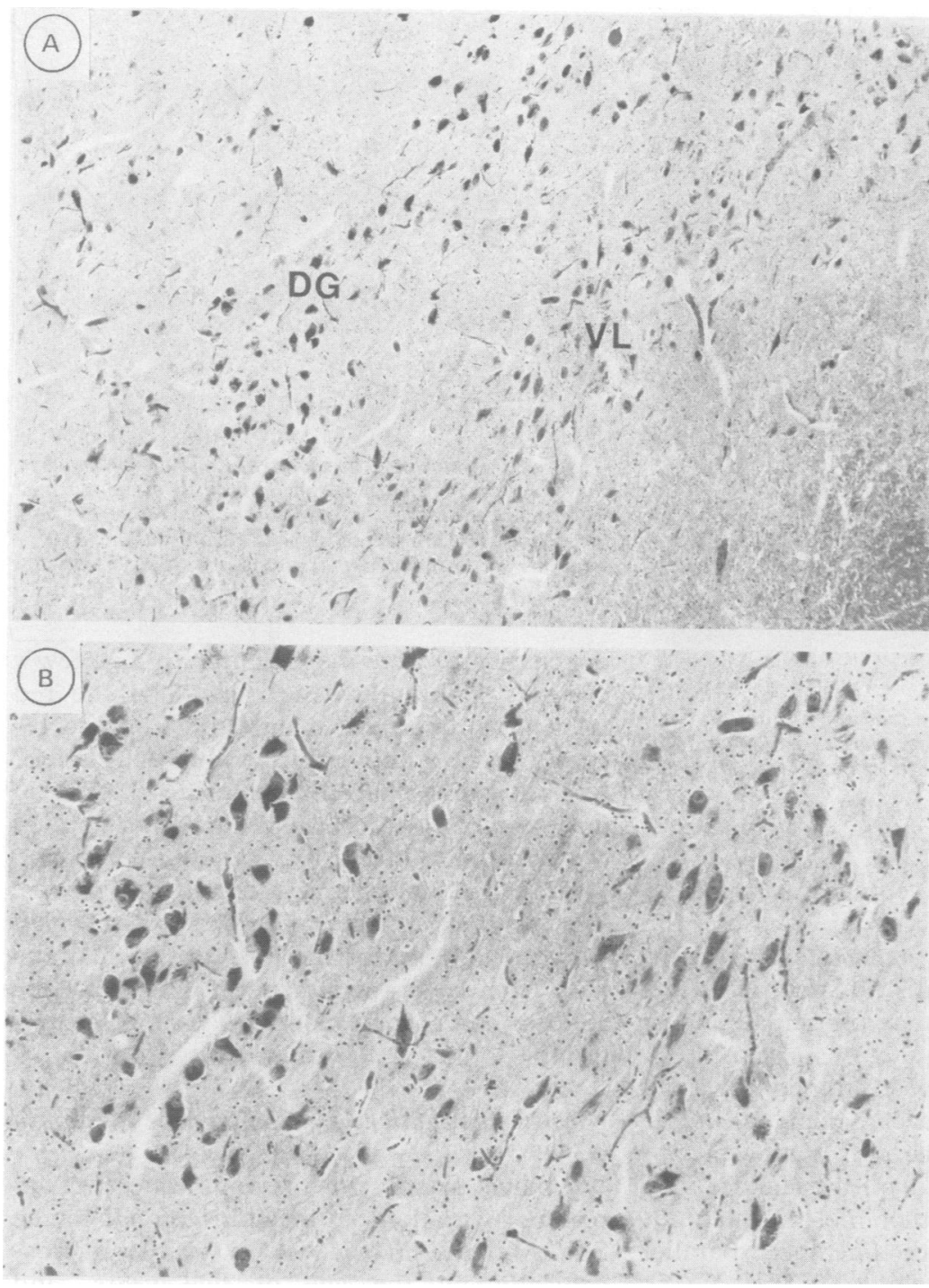

Figure 3 Section of SN equivalent to level 5 showing parallel dorsal (DG) and ventrolateral groups (VL) with heavy and light pigmentation. $H \mathcal{E} E, a, \times 8 ; b, \times 15$.

group formed a ventral pars compacta tier, in contrast to the remaining heavily pigmented nerve cells which formed a dorsal tier. Significant differences in melanin content between nerve cells in these ventral and dorsal tiers were also found using image analysis (table).

The paranigral nucleus extended most of the rostrocaudal length of the SN. Its nerve cells were small and contained amounts of melanin intermediate between those in the ventral and dorsal tiers of the pars compacta (figs 2a-d).

Tyrosine hydroxylase immunostaining showed no major difference in intensity between cell groups. Dendritic processes from groups of ventral tier nerve cells extended ventrally into the pars reticulata, in contrast with the medial to lateral orientation of nerve cell processes in the dorsal tier (fig 4).

\section{Distribution of neuronal death in Parkinson's} disease

In the six cases of PD the entire $S N$ and paranigral nucleus showed Lewy bodies, nerve cell degeneration, nerve cell loss, and reactive gliosis. Each showed the same selective pattern of cell loss with the most severe cell loss and gliosis present in the ventrolateral group (fig 5). Only occasional remaining cells were found here, and these were usually degenerating. The remaining $S N$ and paranigral nucleus contained scattered cells, with clustering persisting in intermediate and dorsal groups. The lightly pigmented nerve cells of the ventral tier were therefore more severely affected than the heavily pigmented dorsal tier. Damage to the paranigral nucleus was of intermediate severity.

Distribution of Lewy bodies in incidental cases The incidental cases were separated into three groups, mild, moderate and severe, on the basis of the severity of nerve cell loss and gliosis (fig 6). Lewy bodies were used as markers of cell degeneration. The mild cases showed Lewy bodies in poorly pigmented cells of the ventrolateral group. In moderate cases some Lewy bodies were also found in the dorsal tier and paranigral nucleus, and in severe cases the pattern of Lewy bodies and nerve cell loss closely resembled that in PD. Cell degeneration, cell loss and gliosis mirrored the same pattern as the Lewy bodies. Thus in successive stages of the disease Lewy bodies and cell degeneration involved the ventral tier of lightly melanised cells, followed by the dorsal tier of heavily melanised cells, with the paranigral nucleus possibly holding an intermediate position.

\section{Discussion}

Normal anatomy of the substantia nigra

Hassler has provided the most comprehensive study of the internal substructure of the SN. His midbrain sections were cut in frontal and horizontal planes, although the latter was sagittally orientated (fig 7). Hassler devised a complex nomenclature for the $\mathrm{SN}$, dividing it into anterior and posterior main parts, and defining 21 subgroups (fig 8). ${ }^{4}$ Some subgroups are consistent and circumscribed and others are arbitrary divisions of cell populations. In view of the highly detailed nature of Hassler's study, and variation between control subjects, the subgroup anatomy cannot be fully abstracted to that seen in transverse sections described in this paper. At level 2 of our study, the paranigral nucleus corresponds approximately to Hassler's medial part of Sam, and our rostral group to the three subdivisions of Sai. At level 5

Table Surface area of melanised neurons occupied by melanin in ventral and dorsal tiers of the pars compacta in control subjects

\begin{tabular}{lll}
\hline & \multicolumn{2}{l}{$\begin{array}{l}\text { Proportion of cell surface area (with SE of } \\
\text { mean) occupied by melanin }\end{array}$} \\
\cline { 2 - 3 } & ventral & dorsal \\
\hline Case 1 & $0.28(0.025)$ & $0.51(0.024)$ \\
Case 2 & $0.18(0.015)$ & $0.57(0.024)$ \\
Case 3 & $0.20(0.018)$ & $0.48(0.027)$ \\
\hline
\end{tabular}

Proportions are derived from the means of 100 melanin and cell surface area measurements $\left(\mu \mathrm{m}^{3}\right)$ in three control cases. In each case the difference between ventral and dorsal tier values
in highly significant $(\mathrm{p}<0.001)$. 


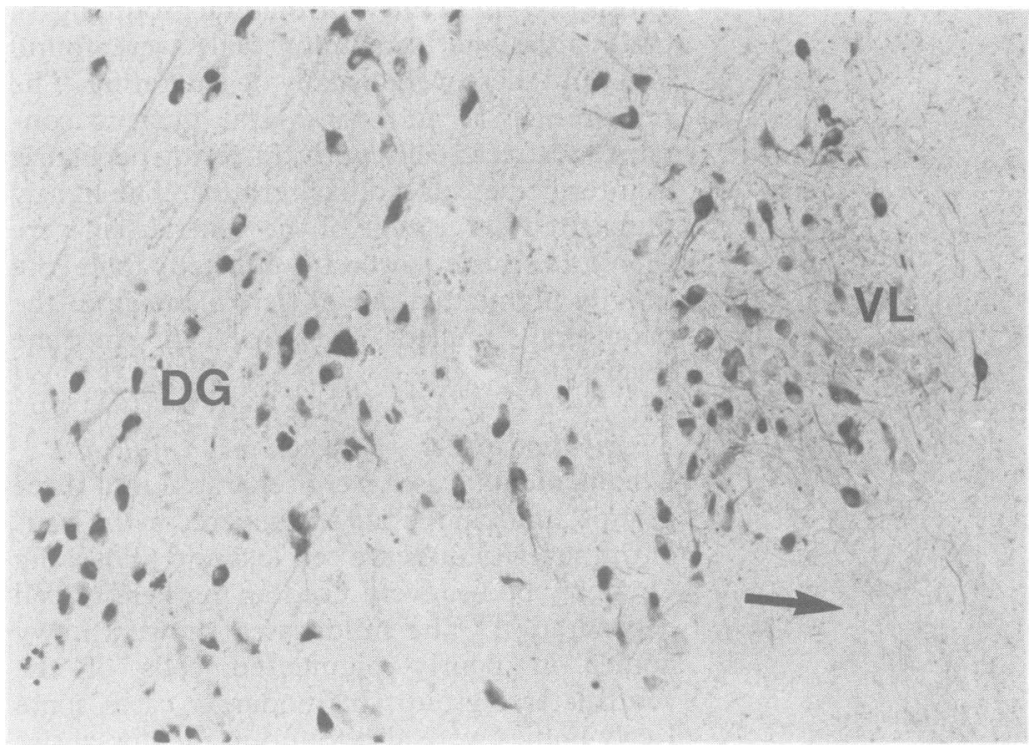

Figure 4 Section stained with tyrosine hydroxylase antiserum showing cells of the dorsal (DG) and ventrolateral groups (VL). The VL group has more closely packed processes which stream ventrally (arrow), in contrast to dendrites of the DG group which are orientated in the medial to lateral direction, $\times 18$.

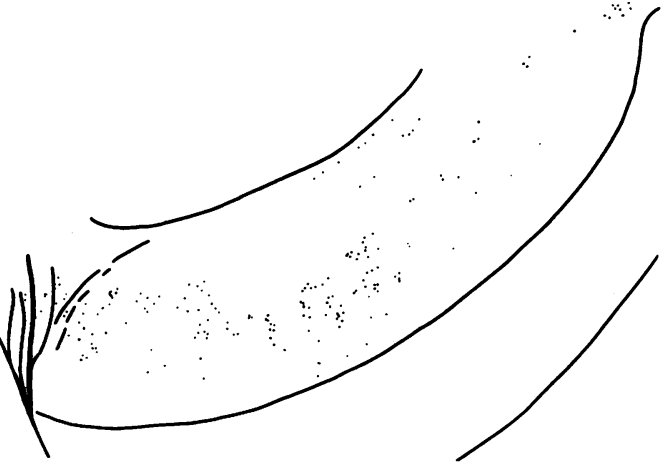
Recently Braak and Braak ${ }^{6}$ using thick (800 between the dorsal and ventrolateral groups. between the anterior and posterior parts of the pars compacta, outlined by Hassler, ${ }^{4}$ the anterior part corresponding to our rostral cell formation at level 8 .

Olszewski and Baxter ${ }^{5}$ examined the substantia nigra in the transverse plane, as in our study, and segregated the pars compacta into parallel divisions $\alpha, \beta$ and $\gamma$. Like Hassler they used Nissl staining so that melanin was not adequately demonstrated. The three parallel divisions are best seen in plates XXXVI, XXXVIII, and XL at the level of the oculomotor nerve, corresponding to our level 5. Cells of the $\alpha$ part, analogous to our ventrolateral group, were thought to form a large population fixed in position. Cells of the $\beta$ part, analogous to our
Normal distribution of melanin in the substantia nigra

We have found no previous reference to regional variations of melanin content in the $\mathrm{SN}$. Melanin in the $\mathrm{SN}$ is visible microscopically by the age of five years, and accumulates progressively with age. Reasonable amounts are found in adolescence, but substantially larger quantities are present by the fifth decade, and more again by the ninth decade. ${ }^{7}$ There is probably no major variation
Figure 6 Distribution diagram of neuronal melanin in the pars compacta in incidental Lewy body disease at level 5. Parts $6 a, b$ and $c$ represent mild, moderate and severe cases. The large dots indicate the

distribution of Lewy bodies. Each large do represents a single Lewy body present in equivalent position in one

representative section from each of four or five cases for each illustration.
(A)

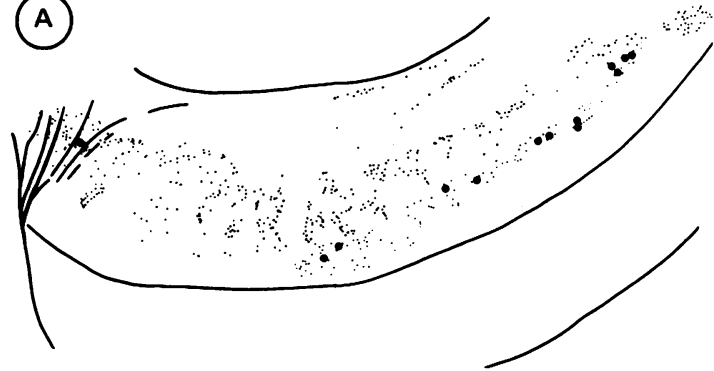

(c)

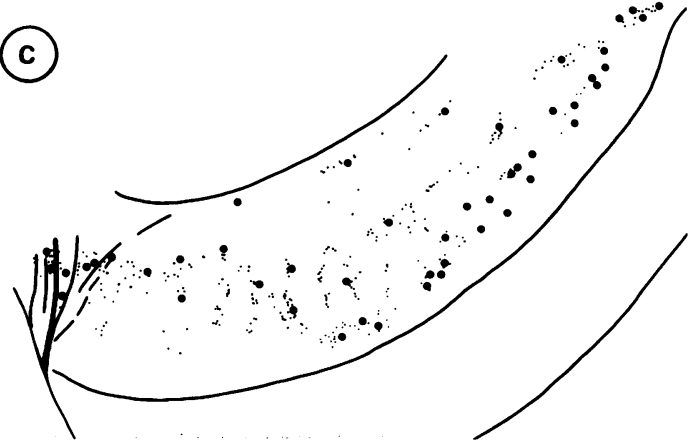

Figure 5 Diagrammatic representation at level 5 (as in fig $1 e$ ) of the distribution of melanin in surviving pars compacta cells in the $S N$ in $P D$. The ventrolateral group is absent, there are few cells in the paranigral nucleus, and intermediate and dorsal groups are greatly reduced.

dorsal group, were more scattered. The few $\gamma$ cells lay adjacent to the capsule of the red nucleus as described in this study, and correspond to the additional $\mathrm{hJ}_{1}$ group of Hassler. ${ }^{4}$ $\mu \mathrm{m})$ sections in the transverse plane, applied the term pars diffusa for scattered cells lying They emphasised the largely arbitrary division group.

(B)

(B)

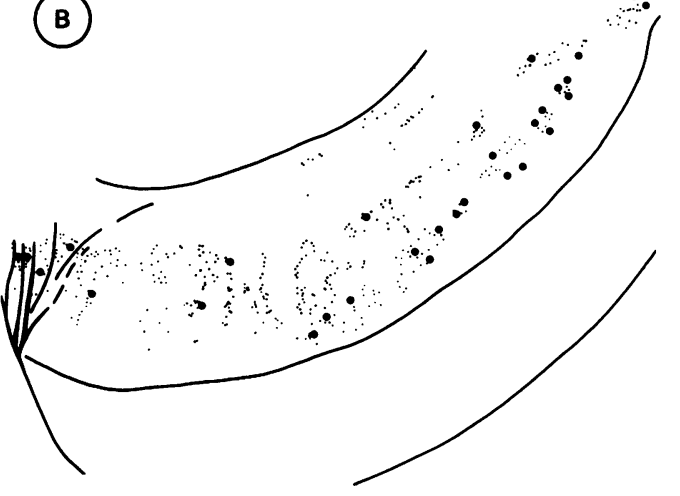


Figure 7 Outline of the brainstem bisected by a vertical line representing Hassler's frontal plane. represents Hassler's "horizontal" plane, which was orientated to the sagittal axis, thus running parallel to a line passing from the oculomotor nerve medially sloping caudally to the pars lateralis laterally. It is not possible to determine the exact ventrodorsal plane of these "horizontal" sections. The more oblique line running from the oculomotor nerve ventrally to the superior colliculus dorsally indicates the plane of section used in this study, perpendicular to the axis of the brainstem. The broad horizontal line

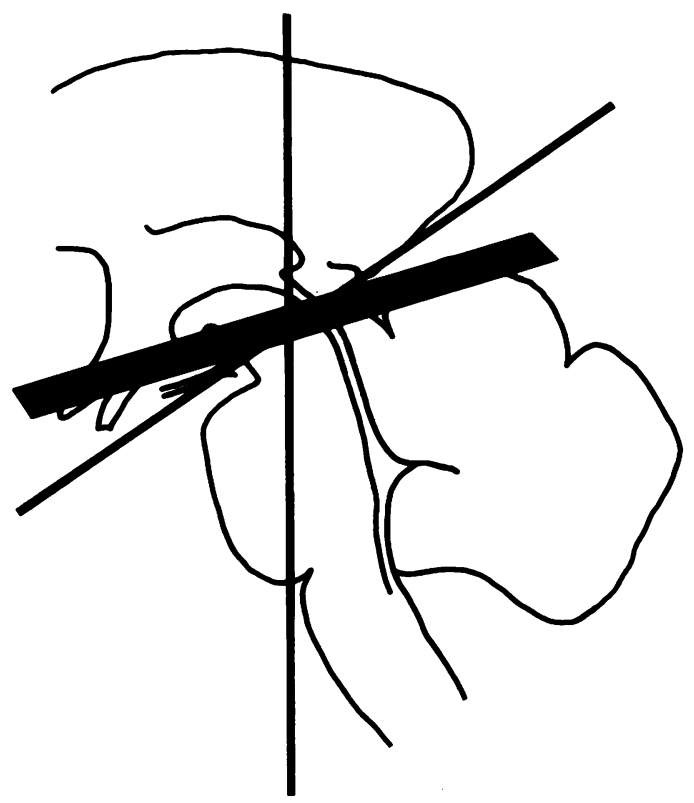

between individuals in the amount of melanin at a given age, and apparent variations in melanin content have only rarely been reported. ${ }^{8}$

We identified two main populations of melanised neurons in the SN. Heavily pigmented neurons formed the rostral, dorsal, intermediate and caudal groups. Lightly pigmented neurons made up the ventrolateral group. These two main populations formed dorsal and ventral tiers or plates, the dorsal tier extending further in rostral and caudal directions, and in the medial to lateral plane. Thus on passing from the rostral to caudal SN the dorsal tier was established first, followed by a dominant ventral tier in the mid-SN, which then vanished caudally in preference to the dorsal tier. The tiers of heavily and lightly pigmented cells of the dorsal and ventrolateral groups lay parallel

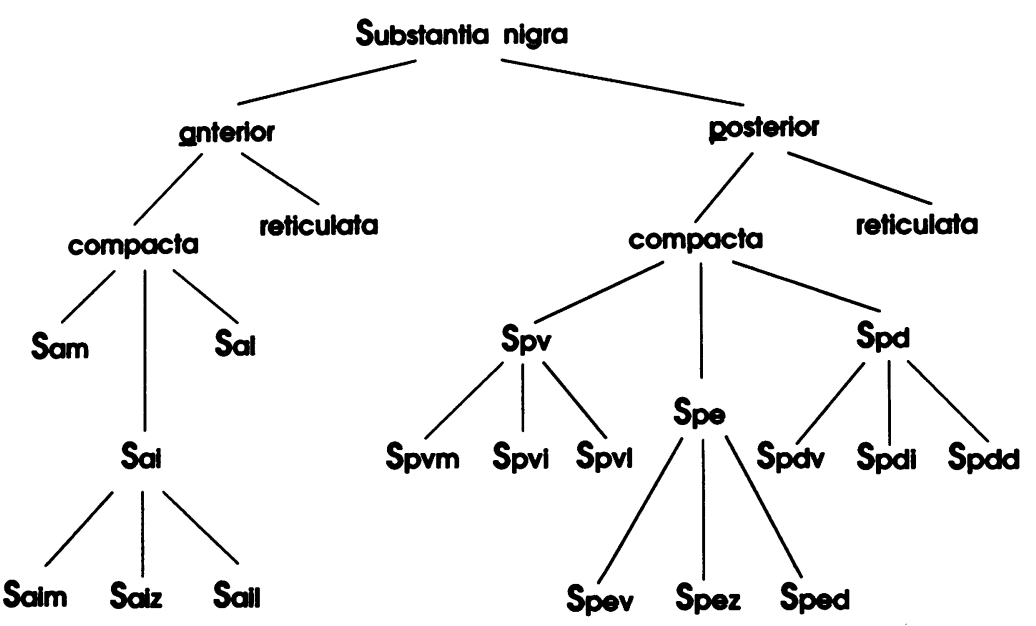

Figure 8 Hassler's subdivisions of the SN. Sai forms the main ventral and medial group, and Spd the main dorsal and lateral group. Spe corresponds mostly to our ventrolateral group, and Spv to our intermediate group. Saivz and Saivl are two additional ventral groups located lateral and a little dorsal to the three main subgroups of Sai. Spzz and Spzv are two extra cell areas lying centrally, and Spcd is the pars lateralis. Spcg is another group within Spev. The $e J$ group is an extra clump of cells between Spdd and Sped, and the J, group refers to additional cells which lie adjacent to the capsule of the red nucleus. The abbreviations are $S$ for substantia nigra, a or $p$ for the anterior or posterior division, and $m$ for medial, $i$ intermediate, l lateral, $z$ central, $v$ ventral, e external, $d$ dorsal, $g$ group.

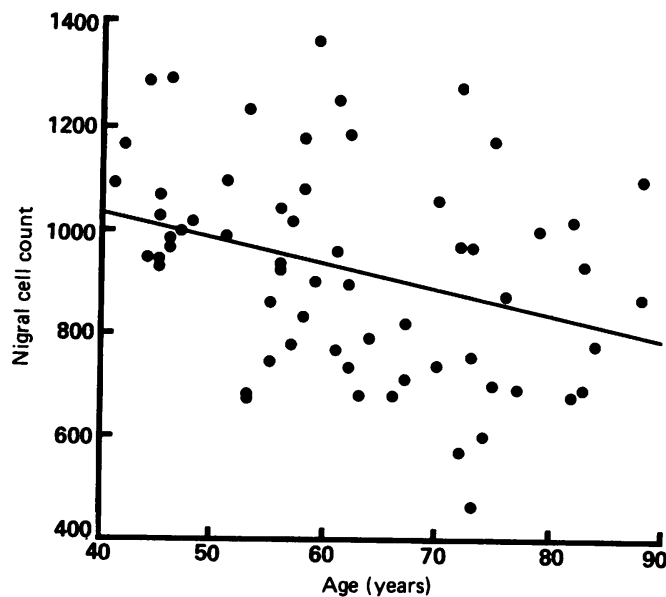

Figure 9 Counts of pigmented cells in complete horizontal sections of $S N$ (includes dorsal and ventral tiers) and paranigral nucleus in controls.

to each other. Dark and light cells were closely apposed where there were bridging cell columns, and in the lateral $\mathrm{SN}$ where contrasting cell groups were often juxtaposed. Cells of the paranigral nucleus were smaller than those in the $S N$, and contained relative amounts of melanin intermediate between that in ventral and dorsal tiers.

\section{Distribution of neuronal death in Parkinson's} disease and incidental cases

Hassler's second main publication on the SN concerned the pattern of neuronal loss in PD. 9 He noted differential damage in the $S N$ pars compacta with complete destruction of groups Spez, Sped and Spedd, corresponding to the ventrolateral group. Spev of the intermediate group was often destroyed, and cells of $\operatorname{Sam} \beta$, Spvl and Spvi were three quarters lost. The dorsal $\mathrm{SN}$ and pars lateralis were relatively preserved. Hassler pointed out that the nigral lesion of post-encephalitic Parkinson syndrome was, in contrast, very severe and nonselective. Similar findings were acknowledged by later authors, ${ }^{1011}$ and comparable diagrams were used to illustrate their observations..$^{12}$

Our observations on the pattern of cell death in PD are similar to those of Hassler, although we could not identify multiple grades of damage between subpopulations. We found complete neuronal loss in the ventrolateral group, and moderate numbers of preserved cells scattered in the dorsal pars compacta and pars lateralis. This differential vulnerability was emphasised by the findings in incidental Lewy body disease. Lewy bodies and neuronal degeneration spread from the ventrolateral part to other regions of the pars compacta.

Recently Hirsch $e t$ al ${ }^{13}$ described a study of five populations of tyrosine hyroxylase positive midbrain neurons in PD and controls; the SN pars compacta and pars lateralis, the central grey substance, A8 and A10 cell groups. The study was not comparable with ours as the same subpopulations of the pars compacta were not examined. They found that between these populations melanised rather than nonmelanised cells were preferentially lost. Within each of the five populations non-melanised 
cells were relatively preserved compared with melanised cells, but there are very few $(16 \%$ of total) non-melanised cells in the pars compacta, and differential vulnerability may not be determined simply by the presence or absence of melanin. The study was interpreted as supporting melanin as conferring selective vulnerability in midbrain tyrosine hydroxylase containing neurons. However, no correlation was found in the locus coeruleus and nonmelanised neuronal populations are damaged in PD.

Our results differ from those of Mann and Yates, who found that in eight cases of PD remaining nigral cells had $15 \%$ less pigment than age-matched controls. ${ }^{1}$ Their study design was also not comparable. The comparison between controls and PD probably utilised dorsal tier neurons in view of the near complete loss of ventral tier cells in PD. Despite their results achieving a level of significance there was considerable overlap. It remains possible that within the dorsal tier of the $\mathrm{SN}$ heavily pigmented cells are lost in preference to lightly pigmented cells.

\section{Distribution of neuronal death in ageing}

The physical burden of melanin is believed to account for loss of pigmented neurons in the $\mathrm{SN}$ in middle and late age, ${ }^{7}$ but the speed of this process is unclear. Estimates of cell depletion have varied from $9-25 \%$ per decade in comparatively small studies totalling 85 subjects, in which degenerative disease was not always excluded. ${ }^{14-16}$ Such figures have suggested that PD might result from the combination of an early life insult to the $\mathrm{SN}$, causing serious cell depletion but no symptoms, combined with age-related neuronal attrition over subsequent years leading to the onset of symptoms. We have counted pigmented nigral cells in unilateral horizontal $\mathrm{H} \& \mathrm{E}$-stained sections of the mid-SN in 62 persons, aged 40 to 90 years, without degenerative disease or Lewy bodies in the SN. ${ }^{17}$ A considerable scatter of cell counts reflected minor variations between section levels and between cases. There was an apparently linear decrease in counts (correlation coefficient, $\mathrm{r}=0.34, \mathrm{p}<0.01$ ) with a loss of $4.7 \%$ to $6.0 \%$ of cells per decade from the fifth to ninth decades (fig 9). Extraneuronal melanin, used as a marker of cell death, was not observed in 23 persons aged eight to 39 years. Another recent study used $\alpha$-dihydrotetrabenazine as a marker of dopaminergic innervation in the caudate nucleus in 49 subjects aged 65 to 95 years. There was a linear fall in binding of this ligand, with a wide scatter, amounting to $7 \cdot 4 \%$ per decade of the extrapolated level at birth. ${ }^{18}$

These results do not support the view that ageing contributes significantly to the evolution of PD, because at onset of PD the approximate $\mathrm{SN}$ cell loss is $60 \%$ and striatal dopamine loss $80 \% .^{12}{ }^{17}$ In addition age-related cell loss is likely to preferentially affect heavily rather than lightly pigmented neurons which are situated in the dorsomedial $\mathrm{SN}$, an area that projects preferentially to the caudate nucleus. ${ }^{19}$ The study of Scherman $e t$ al $^{18}$ is consistent with this notion because there was a slower fall in $\alpha$ dihydrotetrabenazine binding in the putamen in ageing, which was the converse of PD. In contrast the ventrolateral $\mathrm{SN}$ projecting to putamen, especially caudally, is preferentially damaged in PD, whereas in the caudate nucleus only the dorsal rostral part is severely depleted, with other areas having substantial levels. ${ }^{20}$

MPTP (1-methyl-4-phenyl-1,2,3,6-tetrahydropyridine)

MPTP has low affinity for synthetic and retinal melanins, and its active metabolite MPP + has high affinity for synthetic melanin and neuromelanin. ${ }^{21}$ In primates damage to the substantia nigra appears to be greatest in parts containing the most melanin, and chloroquine can protect against MPTP-damage because its high affinity for melanin prevents MPP + binding. ${ }^{22}$ Consequently if MPTP shows regional damage in humans the dorsal tier would be expected to be more affected than the ventral tier. The evidence available suggests that MPTP does not cause the same regional damage to the $\mathrm{SN}$ as seen in PD, but comparatively acute models are available only. Rhesus monkeys given MPTP show severe and equal reductions of dopamine in the caudate nucleus and putamen, ${ }^{23}$ unlike PD where caudate dopamine is relatively preserved. ${ }^{12}$

\section{Substantia nigra and striatal subdivisions}

It is considered likely that subpopulations of dopaminergic midbrain neurons project either to striosome or matrix compartments of the striatum, ${ }^{24}$ which differ in their ontogeny, neurochemical features and extrastriatal connections. There are approximate anteriorposterior ${ }^{19}{ }^{25-28}$ and medial-lateral relationships between the $\mathrm{SN}$ and striatum, but the dorsal to ventral relationship is inverted. ${ }^{1926}$ Thus the ventral pars compacta projects to the dorsal caudate, and the dorsal pars compacta to the ventral caudate. In the monkey there is a predominant rostral and dorsal $\mathrm{SN}$ population projecting to caudate and a caudal and ventral population projecting to putamen. ${ }^{29}$ In humans this relationship is reflected by a greater loss of dopamine in the putamen compared with the caudate nucleus in PD, ${ }^{12}$ and the ventral-dorsal inversion by a greater loss in the dorsal striatum, regional patterns which correspond to the severe neuronal loss in the ventral $\mathrm{SN} .^{20}$ In the cat, rat and monkey the cell dense part of the SN (A9) may be analogous to the ventrolateral group. ${ }^{30-33}$ In this study nerve cell processes of the ventral tier were ventrally directed corresponding to the arrangement seen in the rat. ${ }^{34}$ This ventral tier predominantly innervates striosomes, whereas the dorsal cell sparse part (A8) and the ventral tegmental area (A10) predominantly innervates matrix. ${ }^{30-33}$ Additionally the calcium binding protein, calbindin, is detectable in rat dorsal tier neurons and in projections to matrix, but not in ventral tier neurons and striosomes. ${ }^{34}$

The difference in melanin content between ventral and dorsal tier pars compacta neurons provides evidence for their functional disparity, because melanin production depends on 
the auto-oxidation of dopamine. A low melanin content could be due to reduced dopamine synthesis or more efficient transport to the nerve terminal, but the dopamine turnover rate is lower in striosomes supporting the first of these possibilities. ${ }^{35}$ The lower rates of dopamine turnover and release from nigral neurons projecting to striosomes corresponds to the more efficient presynaptic dopamine uptake in matrix compared with striosomes. The evidence points to functionally distinct ventral and dorsal tier neurons projecting to striosomes and matrix respectively. ${ }^{36}$

Mechanisms of cell death in Parkinson's disease In ageing, heavily melanised dorsal tier nigrostriatal neurons are more susceptible than ventral tier neurons, and melanin may also be a weak factor prompting neuronal death in $\mathrm{PD}^{13}$ and MPTP-induced Parkinsonism. In addition MPTP in the dog produces greatest degeneration of nigrostriatal terminals in the matrix zone in the anterior caudate nucleus, ${ }^{37}$ and this may be due to enhanced uptake of MPP + in the matrix compared with striosomes. ${ }^{36}$ Thus although the main cause of MPTP-induced neuronal degeneration is thought to be mitochondrial complex I inhibition, regional variations in dopamine-uptake and melanin may contribute to selective neurotoxicity. The predominant pattern of neuronal loss caused by MPTP does not mirror that of PD, but prolonged low level exposure would be required to emulate the timecourse of PD. The differential neuronal loss of PD is not unique to the disease because we have observed the same pattern of neuronal loss, associated with Lewy bodies, in a case of dopa-responsive dystonia.$^{38}$ In this case the differential damage was even more striking with complete cell loss in the ventral tier and normal populations elsewhere. This difference suggests important variations in neuronal metabolism which relate to pathogenesis.

Most other nigral degenerations do not show this pattern. For example, similar degrees of ventral and dorsal tier neuronal loss in SteeleRichardson-Olszewski disease are reflected by similar reductions in caudate nucleus and putamen dopamine. ${ }^{39}$ However, in cases of striatonigral degeneration without complete nigral cell loss there is also relative preservation of dorsal tier neurons, ${ }^{4041}$ and this is reflected by lower dopamine levels in putamen than caudate nucleus. ${ }^{42}$ Thus the ventral-dorsal tier differential cell loss is not exclusive to neuronal degenerations associated with Lewy bodies, but imply a common pathogenetic mechanism in these disorders.

Ventral tier cells could be relatively vulnerable or dorsal tier cells relatively resistant to this kind of injury. The case of dopa-responsive dystonia had an abnormally low melanin content in the surviving dorsal tier and paranigral nucleus suggesting that catecholamine metabolism might be abnormal..$^{38}$ Indeed, the contrasting melanin content of these tiers correlates with their differential vulnerability, although the probably lower dopamine turnover rate in the susceptible ventral tier is against a role for catecholamine metabolism. ${ }^{35}$
Other factors therefore need consideration, such as the differential localisation of metabolic factors which may play a role in the pathogenesis of cell death.

We are grateful to Dr W Poewe for interpreting Hassler's papers, to Professor PL Lantos for contributing control subjects and incidental cases, and to Dr PJ Luthert for assisting with image analysis. Dr Gibb was a Medical Research Council Training Fellow when this work was carried out.

1 Mann DMA, Yates PO. Possible role of neuromelanin in the pathogenesis of Parkinson's disease. Mech Ageing Dev 1983;21:193-203.

2 D'Amato RJ, Alexander GM, Schwartzman RJ, Kitt CA Price DL, Snyder SH. Evidence for neuromelanin involvement in MPTP-induced neurotoxicity. Nature involvement in

3 Gibb WRG, Lees AJ. The relevance of the Lewy body to the pathogenesis of idiopathic Parkinson's disease. J Neurol Neurosurg Psychiatry 1988;51:745-52.

4 Hassler R. Zur Normalanatomie de Substantia nigra. $J$ Psychol Neurol 1937;48:1-55.

5 Olszewski J, Baxter D. Cytoarchitecture of the human brain stem. Basel: S Karger, 1954.

6 Braak H, Braak E. Nuclear configuration and neuronal types of the nucleus niger in the brain of the human adult. Hum Neurobiol 1986;5:71-82.

7 Mann DMA, Yates PO. Lipoprotein pigments-their relationship to ageing in the human nervous system. II.
The melanin content of pigmented nerve cells. Brain 1974;97:489-98.

8 Spence AM, Gilles FH. Underpigmentation of the substantia nigra in chronic disease in children. Neurology 1971;21:386-90.

9 Hassler R. Zur Pathologie der Paralysis agitans und des postenzephalitischen Parkinsonismus. J Psychol Neurol 1938;48:387-455

10 Buttlar-Brentano K. Das Parkinsonsyndrome im Lichte der lebensgeschichtlichen Veranderungen des Nucleus basalis. J Hirnforsch 1955;22:55-76.

11 Greenfield JG, Bosanquet FD. The brain-stem lesions in parkinsonism. J Neurol Neurosurg Psychiatry 1953;16:213-26.

12 Bernheimer H, Birkmayer W, Hornykiewicz O, Jellinger $\mathrm{K}$, Seitelberger $F$. Brain dopamine and the syndromes of Parkinson and Huntington. J Neurol Sci 1973;20:415-55.

13 Hirsch E, Graybiel AM, Agid YA. Melanized dopaminergic neurons are differentially susceptible to degeneration in Parkinson's disease. Nature 1988;334:345-8.

14 McGeer PL, McGeer EG, Suzuki JS. Aging and extrapyramidal function. Arch Neurol 1977;34:33-5.

15 Hirai S. Ageing of the substantia nigra. Adv Neurol Sci 1968;12:845-9.

16 Mann DMA, Yates PO. Pathological basis for neurotransmitter changes in Parkinson's disease. Neuropath Appl Neurobiol 1983;9:3-19.

17 Gibb WRG. The significance of the Lewy body in the diagnosis, epidemiology and pathogenesis of idiopathic Parkinson's disease. Thesis, University of London, 1987.

18 Scherman D, Desnos C, Darchen F, Pollak P, Javoy-Agid F, Agid Y. Striatal dopamine deficiency in Parkinson's disease: role of aging. Ann Neurol 1989;26:551-7.

19 Szabo J. Organization of the ascending striatal afferents in monkeys. J Comp Neurol 1980;189:307-21.

20 Kish SJ, Shannak K, Hornykiewicz O. Uneven pattern of dopamine loss in the striatum of patients with idiopathic Parkinson's disease. New Engl J Med 1988;318:876-80.

21 Snyder SH, D'Amato RJ. MPTP: a neurotoxin relevant to the pathophysiology of Parkinson's disease. Neurology 1986;36:250-8.

22 D'Amato RJ, Lipman ZP, Snyder SH. Selectivity of the parkinsonian neurotoxin MPTP: toxic metabolite MPP + binds to neuromelanin. Science 1986;231:987-9.

$23 \mathrm{Pifl} C \mathrm{Ch}$, Schingnitz G, Hornykiewicz $\mathrm{O}$. The neurotoxin MPTP does not reproduce in the rhesus monkey the interregional pattern of striatal dopamine loss typical of human idiopathic Parkinson's disease. Neurosci Lett 1988;92:228-33.

24 Graybiel AM. Dopaminergic and cholinergic systems in the striatum. In: Crossman AR, Sambrook MA, eds. Neural mechanisms in disorders of movement. London: John Libbey, 1989:3-15.

25 Bédard P, Larochelle L, Parent A, Poirier LJ. A correlative study based on neuroanatomical and neurochemical criteria in the cat and monkey. Exp Neurol 1969;25: 365-77.

26 Carpenter MB, Peter P. Nigrostriatal and nigrothalamic fibers in the rhesus monkey. J Comp Neurol 1972;144:

27 Fallon JH, Moore RY. Catecholamine innervation of the basal forebrain. IV. Topography of the dopamine projection to the basal forebrain and neostriatum. J Comp Neurol 1978;180:545-80.

28 Gerfen CR. The neostriatal mosaic. I. Compartmental organization of projections from the striatum to the substantia nigra in the rat. J Comp Neurol 1985;236: 454-76.

29 Smith Y, Parent A. Differential connections of caudate 
nucleus and putamen in the squirrel monkey (Saimiri sciureus). Neuroscience 1986;18:347-71.

30 Jimenez-Castellanos J, Graybiel AM. Subdivisions of the dopamine-containing A8-A9-A10 complex identified by their differential mesostriatal innervation of striosomes and extrastriosomal matrix. Neuroscience 1987;23:223-42.

31 Herkenham M, Moon-Edley S, Stuart J. Cell clusters in the nucleus accumbens of the rat, and the mosaic relationship of opiate receptors, acetylcholinesterase and subcor

32 Gerfen CR, Herkenham M, Thibault J. The neostriatal matrix: II. Patch- and matrix-directed mesostriatal dopaminergic and non-dopaminergic system. $J$ Neurosci 1987;7:3915-34.

33 Langer LF, Graybiel AM. Distinct nigrostriatal projection systems innervate striosomes and matrix in the primate striatum. Brain Res 1989;498:344-50.

34 Gerfen CR, Baimbridge KG, Thibault J. The neostriatal mosaic: III Biochemical and developmental dissociation mosac. III. Biochernical and developmental dissociation of patch-matrix

35 Fuxe K, Andersson K, Schwarcz R, et al. Studies on different types of dopamine nerve terminals in the different types of dopamine nerve terminals in the
forebrain and their possible interactions with hormones and with neurons containing GABA, glutamate, and opioid peptides. Adv Neurol 1979;24:199-215.

36 Graybiel AM, Moratall R. Dopamine uptake sites in the striatum are distributed differentially in striosome and matrix compartments. Proc Natl Acad $S c i$ US 1989;86:9020-4.

37 Turner BH, Wilson JS, McKenzie JC, Richtand N. MPTP produces a pattern of nigrostriatal degeneration which coincides with the mosaic organization of the caudate nucleus. Brain Res 1988;473:60-4

38 Gibb WRG, Narabayashi H, Yokochi M, Iizuka R Additional pathological observations in juvenile onset parkinsonism with dystonia. Neurology 1989;39 (suppl) 1:139.

39 Ruberg M, Javoy-Agid F, Hirsch E, et al. Dopaminergic and cholinergic lesions in progressive supranuclear palsy. Ann Neurol 1985;18:523-9.

40 Adams RD, van Bogaert L, Eecken HV. Striato-nigra degeneration. J Neuropath Exp Neurol 1964;24:584-608.

41 Fearnley JM, Lees AI. Striatonigral degeneration. A clinicopathological study. Brain 1990;113:1823-42.

42 Spokes EG, Bannister R, Oppenheimer DR. Multiple system atrophy with autonomic failure. $J$ Neurol $S c i$ 1979;43:59-82. 